\title{
“The Effectiveness' of Total Quality Management In Service Quality of Ministry of Health and Child Care"
}

\author{
Gore Sekai Noreen ${ }^{1}$, Sithole Kudakwashe ${ }^{2}$, Gondo Kudzanayi ${ }^{3}$ \\ ${ }^{1-3}$ Faculty of Commerce and Law, Zimbabwe Open University, Harare, Zimbabwe
}

\begin{abstract}
Causes of child death were identified; strategies and policies were put in place to help minimize child death. The challenge is that the service quality is being ignored which is leading to more child deaths. . The research sought to address the gap caused by lack of quality service in Prevention of Mother to Child Transmission ( PMTCT). The study survey was restricted to 14 health institutions in Chikomba District. The health institutions have 217 staff who could participate. According to PMTCT Service statistics $3^{\text {rd }}$ quarter 2012 for Chikomba District the beneficiaries totaled 1185. Four Chiefs in the District and two relevant stakeholders District Aids Coordinator (DAAC) Focal Officer and PMTCT Coordinator Elizabeth Glaser Pediatric Aids Foundation (EGPAF) were also part of the target population. All these added would give a total target population of 1407 . The sample size comprised of 98(45\%) participants from the 14 health institutions, 532 beneficiaries , 2 stakeholders and 4 Chiefs . 28 questionnaires were distributed to management, 70 to employees and 2 to (DAAC) and (EGPAF) representatives. Focus group discussions were carried out on 532 beneficiaries and 4 community leadership. Interviews were done with 14 senior manager, 4 community leaders and 2 stakeholder representatives. We found out that there are eight practices that support total quality management (TQM) positively; these are top-management commitment, teamwork and participation, process management, customer focus and satisfaction, resource management, organization behavior and culture, continuous improvement, and training. In conclusion we found out that for successful TQM implementation employee involvement, senior management commitment, training and also taking into account the needs of external and internal customers is very important. There is need to carry out further research on this area so that relevant frameworks are built.
\end{abstract}

Key Words: TQM -Total Quality Management, PMTCT -Prevention of mother to child, health sector, service quality

\section{BACKGROUND TO THE STUDY}

The PMTCT programme was launched in 1999 under the framework of the national HIV and AIDS policy of 1999. These services were integrated into the antenatal care services. According to the PMTCT report of 2008, comprehensive PMTCT services in Zimbabwe include routine HIV testing of pregnant women and provision of ARV prophylaxis (initially based on single dose Nevirapine to reduce mother-to-child transmission of HIV). PMTCT services are currently offered in over 910 health facilities with a further 650 sites providing routine antenatal and postnatal care but unable to provide on-site HIV testing. Throughout 2008, 224,637 pregnant women booked for a first ANC visit. Of these, 226,713 pregnant women were counseled for HIV and $78 \%$ were tested. The overall ANC HIV prevalence was $15.6 \%$ in 2009 . Uptake of single dose Nevirapine (sdNVP) by HIV-positive pregnant women at ANC sites is currently 91\%, with over 24,896 HIV-positive pregnant women receiving prophylaxis. Owing to the limited effectiveness of this regimen, the More Efficacious Regimen (MER) was piloted and is in the process of being rolled out along with an urgent emphasis on treating those pregnant women with HIV who require it for their own health. Based on the national HIV estimates from 2008/2009, it was estimated that at a population level, 50,702 pregnant women were expected to be HIV-positive. The national PMTCT programme was therefore able to achieve an estimated $49 \%$ coverage of PMTCT interventions in 2009. This is far below the expected universal coverage of PMTCT services given that about $95 \%$ of HIV in children is through vertical transmission. A number of challenges face the PMTCT programme including low human resource capacity, low male partner participation, low rate of HIV status disclosureIn order to effectively reduce the childhood mortality trends in the country, there is need to relook at the quality of services being provided at the health centres, there is need to a outlining the major target killers, look at the intervention strategies and actions and check the quality of the strategies.

\subsection{Statement of the problem}

The infant mortality rate is not decreasing with the expected rate. A lot strategies have been put in place to reduce infant mortality rate for example PMTCT programme but still it is not decreasing with the expected rate. 


\subsection{Research Questions}

The research was carried out with the intention of finding answers to the following questions;

1. How comparable is the implementation of TQM in the Zimbabwean public health institution to international best practices?

2. What improvements can be done on TQM in PMTCT programmes?

\section{LITERATURE REVIEW}

\subsection{Quality Assurance.}

Bin Seed (1994, pp. 30), carried out a study to determine the attitudes of nurses towards the effectiveness of QA programmes in the $\mathrm{MOH}$ hospitals and the factors that affect the effectiveness of these programmes. The field study was conducted in six out of fourteen hospitals that apply such programmes. The researcher used a questionnaire that had been distributed to 646 people. The researcher tested a number of hypotheses using the Chi square (X2) test statistics. The conclusions of the study were as follows: Two thirds of the sample was of the opinion that the programmes were effective and one third was of the opinion that the programmes were ineffective. The reasons that negatively affect the effectiveness of the quality programmes in the hospitals include: the different nationalities of the hospital employees, the non-availability of quality specialists, high turnover of employment among the nurses, ignorance of the concept of QA by the top management and a lack of encouragement of the implementation of QA programmes, lack of employee awareness towards the importance of applying QA programmes, their effectiveness and the non-availability of standards and criteria for evaluation.

Our opinion in the finding is that for me the issue of different nationalities itself does not affect the effectiveness of quality programmes, and in Zimbabwe, Public health services sector is dominated by the one Nationality for example Chikomba District does not have any other nationality within the thirty three clinics. In Zimbabwe public health service sector also does not have quality assurance personnel and there is much ignorance of the concept by top management. They also talk about quality but there are no standards and criteria for evaluation of quality. The study was undertaken in $\mathrm{MOH}$ Hospitals and concentrated on the opinions of one category of hospital staff, namely nurses. The results would have been more valid if it had covered employees in other fields within the hospitals.

\subsection{Patient Satisfaction regarding Provision of Health Services}

Berte's (1987, p. 128) study identified demographic factors that make patients select government or private hospitals for treatment The study was applied in two government hospitals and two private hospitals in the city of Riyadh The researcher used a questionnaire. The researcher concluded that most of the citizens who use the government hospitals are of old age, low educational level (a maximum of secondary educational level) and low income. On the other hand, those who use the private hospitals are younger and of higher educational levels and income. Our view is that these findings truly affect the quality of services offered. For example those who have been identified to be visiting government hospitals have low educational levels and they may not be bold enough to express themselves in the case that they were not satisfied by the quality of services offered. Also because of low income they can not say much as that may lead them to be referred to private hospital which will be more expensive.

\subsection{Total commitment and support from central health authorities}

TQM requires top management support.Public health care services in PMTCT operate under constraints of limited resources,high demands and competing health problems. Furthermore, we often find unequal allocation of resources between different levels of facilities, workforce categories andvarious departments. This particularly affects PMTCT services. Often, hospitals and clinics receive financial support or funds directed towards HIV/AIDS programmes. Staff training is insufficient and few unifying professional organisations exist. Particularly at the peripheral level, OI units are understaffed, underutilized and do not receive the importance they deserve. In the scenario described above, quality is an alien word. Central health planners have to work and operate within these given constraints. The first step towards changing the status quo, is for central health planners to define national objectives and display total commitment to a quality transformation process.

\subsection{Provide quality PMTCT services to all service-users}

TQM means service-user satisfaction. In the context of PMTCT services, our customers are patients, physicians, health care workers and epidemiologists. Limited resources might not allow to meet the immediate needs of all service-users. Priorities will have to be set, based on the availability of financial and human 
resources, disease pattern and current health concerns of the population. Service-user satisfaction in this sense means, reliable, reproducible and timely test-results and service delivery in line with organizational objectives. 2.13 Give priority to training and create an environment of continuous learning TQM puts special impetus on training. Well-trained employees make more efficient team members. PMTCT services need a workforce of competent, responsible, autonomous and flexible employees to accomplish organisational objectives and provide the quality of service required. As stated by Green (1994), health services rely heavily on personnel, which often represent up to $75 \%$ of revenue expenditure. Therefore, training and development of PMTCT personnel is the most efficient and cost-effective way of quality improvement. Often identified training needs for PMTCT personnel are to reinforce technical skills and to acquire managerial and organisational skills. Given that resources are limited, special efforts are required to ensure that training resources are effectively used. Health planners have to identify training programmes with minimal financial implications and non-monetary training resources, such as existing staff skills .

\section{METHODOLOGY}

A survey was conducted to collect data from a sample of 14 healthy institutions. The total population was thirty two health institutions in, Chikomba District. The health institution had 217 employees, beneficiaries totaled to 1185, on local leadership the researchers focused on the four Chiefs in the District and 2 relevant stakeholders that is DAAC Focal Officer and PMTCT Coordinator (EGPAF). All these added would give a total target population of 1407. The researcher used the payroll system to draw up the sample frame for employees and for patients PMTCT register was used. Interviews, questionnaires and focus group discussions were administered. The researchers used the thematic, comparative and content analysis methods to analyse the data. For high content validity the questions that were used in the questionnaire, FGD and interviews correlated highly with the studywide questions. Triangulation was also used. Permission was sought from the Ministry of Health and Child Welfare to carry out the research. The researchers also addressed individual concerns and upheld anonymity and confidentiality of all participants.

\section{RESULTS AND ANALYSIS}

From the study findings there is no evidence to suggest that TQM succeeded in the PMTCT programme. About $58 \%$ of the respondents disagreed that TQM was successfully implemented in the programme. It can be said that TQM has not been widely accepted and implemented in the programme. This may be that the implementation part of TQM at the health institutions has not been done in line with the best practices hence it did not succeed. TQM failure may be as a result of different factors that include lack of top management support, resistance to change, the nature of TQM, poor communication, lack of employee involvement among other factors. The implementation of TQM into an organization requires fundamental organizational culture change. Changing an organization's culture is a very difficult task, which often faces resistance. The challenge of implementing TQM is due to the fact that TQM is not a slogan, nor a tool, nor a program; it is an organization paradigm thus implementing TQM might be dealing with replacing, rather than modifying, the organization's culture. It is from this that it may face resistance as some of the employees may be content with the old culture and hence would not be committed or willing to adapt to the new TQM culture. In light with the findings, the PMTCT management may have failed to get employees buy in/commitment to fully implement TQM hence its failure. Brown et al (1994) put forward that the reasons for TQM failure in organisations include among other reasons, poor training, and wasted education and training, inappropriate rewards, failing to transfer true power to employees, outdated business systems and maintaining outdated management practices. The failure of TQM at the health institutions has also been confirmed by the fact that the employees according to majority of the respondents (56\%) has implemented or have been implementing TQM for over 5 years yet TQM has not been applied in all departments. This is in contra with Flood (1993) who argues that TQM is a company-wide effort and hence involves everyone's effort in the organization in order to improve performance. In other words, TQM can be achieved through an integrated effort among personnel at all levels, to increase customer satisfaction by continuously improving performance. The integrated effort among personnel can be achieved by having effective and comprehensive management.

The results also showed that the issue of staff attitude must be considered seriously if we are to improve the service quality of PMTCT programmes. The researcher found out that if the staff have a negative attitude towards the patients the patients are likely not to return again, this will lead to increase of the lost to follow up and defaulters.

\section{CONCLUSIONS}

The study concluded that despite the fact that all the public health institutions are practicing some form of TQM elements, none has matched the international best practices. TQM has not been fully adopted and 
applied in the health sector. The major factors that have hindered the successful adoption of TQM in the PMTCT programme are

- lack of employee involvement,

- lack of training,

- lack of financial resources and

- Lack of senior management involvement in all issues to do with TQM.

\section{RECOMMENDATIONS}

The study presented the following recommendations

i. TQM To be successful, it must be top-management driven and focus on maximising efficiency and effectiveness, through improving systems and processes, as well as aligning business objectives and customer requirements.

ii. The key to implementing TQM is combining a series of philosophies and techniques with practices in the organisation and making sure the total quality management principles are planted into everybody's mind and are run in every department's day-to-day work. The senior managers should first realise the significance of TQM and play an indispensable role in leadership and commitment. They must personally involve themselves in the quality affairs to show their support and stimulate the formation of a companywide quality culture. Training, communication and internal customer needs should be emphasised to increase employee involvement and the coordination of the organisation.

iii. TQM techniques such as JIT should be used to reduce cost and lead time. Defects, errors and breakdown ought to be reduced by every individual, and departments should work towards doing things right at first time. Attention must be paid to all of these requirements in order to satisfy external customer's requirements, which is one of the most important business objectives in the organisation. All these points are worthwhile considering the amount of evidence that successful TQM implementation can positively influence company's performance.

iv. Capacity building

v. Train non health professionals e.g. teachers and religious leaders on counseling and testing by targeting PMTCT services to alleviate the human resource for health crisis

vi. Distribution of supplies such as testing kits and other laboratory supplies, ARV drugs, procurement of protective materials, registration books should be available consistently at heath facility level.

vii. Lobby the government officials on budgeting allocation for health according to MDG call for additional budget for PMTCT.

viii. Promote involvement of development partners in HIV and PMTCT programmes on technical capacity building, tackling stigma and discrimination in the community PMTCT ,logistics management and procurement and additional fund allocation for PMTCT

ix. Decrease the bureaucratic handling of required budget and donor funding so that enough money can find its way to facility level and make it easy to utilise the money.

$\mathrm{x}$. Improve access of information for adolescence, reproductive age woman and community at large by promoting MTCT and PMTCT through mass media

xi. Sustained gender equality and empowerment of women to increase women decision making powering the community on uptake of PMTCT services

\section{REFERENCES}

[1] Berte's (1987 p. 128) Factors affecting patient's choice of hospitals http://www.ncbi.nlm.nih.gov/pubmed/17344716

[2] Brown's 1986 study on 'Social support, self-esteem and depression'. www.ncbi.nlm.nih.gov/../3823299

[3] Green A. (1994). An Introduction to Health Planning in Developing Countries. Oxford, Oxford University Press.

[4] Green A (1994) Total quality management public health laboratories developing countries http://www.phclab.com/images/thesis.pdf

[5] Mallapaty, G (1999) The role of total quality management in raising the service quality of public health laboratories in developing countries www.phclab.com/images/thesis.pdf

[6] Torrington D. \& Weightman J. (1994). Effective Management: People and Organisation, 2nd ed. Hertfordshire, Prentice Hall. 